\title{
Adaptación de cofias metálicas confeccionadas con dos técnicas: cera pérdida colado por centrifugación convencional e inducción
}

Adaptation of metallic copings made with two techniques: loss wax cast conventional centrifugation and induction.

Silvio Over Requena Cisneros ${ }^{1, a ; ~ 4, b}$, Micarla Yanira Loarte Campos ${ }^{2, a ; ~ 4, b}$, Cinthya Cecilia Zelada Ladrón de Guevara ${ }^{3, a ; ~ 4, b}$, Yovana Elisa Flores Valverde 2,a; 4,b, Diana Esmeralda Castillo Andamayo ${ }^{4 a, b, c ; j d, ~ M a r t i ́ n ~}$ Gilberto Quintana del Solar ${ }^{4, a, b, d ; 6 d}$

\section{RESUMEN}

Nuevas técnicas nos están permitiendo migrar de la técnica de cera perdida colado por centrifugado convencional (CPCC) a técnicas como la cera perdida colado por inducción (CPCI). Objetivos: Comparar la discrepancia marginal e interna de cofias unitarias de aleación Cobalto-Cromo (Co-Cr) sobre una línea de terminación chamfer, confeccionadas con dos técnicas: CPCC y CPCI, determinando cual técnica tuvo mejor adaptación marginal e interna. Material y Métodos: Se fabricaron 13 cofias metálicas unitarias de aleación Co-Cr para cada técnica a evaluar. Se utilizó la réplica de silicona para evaluar las discrepancias marginales e internas, cada muestra fue seccionada en cruz en sentido vestíbulo-palatino y en sentido mesio-distal, luego con un estéreomicroscopio se evaluó la zona cervical, axial y oclusal. Resultados: Se evaluó los supuestos de normalidad con la prueba de Shapiro-Wilk. Los análisis estadísticos fueron la prueba t de Student y U Mann-Whitney. La CPCI obtuvo una menor discrepancia marginal e interna en comparación con la CPC pero no se encontró diferencias estadísticamente significativas $(\mathrm{p}>0,05)$ entre ambas técnicas. Conclusiones: Aunque en la mayoría de los puntos de evaluación las cofias realizadas mediante la CPCI presento mejores valores de adaptación marginal e interna en comparación con la CPCC estos resultados sólo son valores descriptivos que no fueron concluyentes, ya que en la mayoría de los puntos evaluados no existió diferencia estadísticamente significativa $(p>0,05)$. Observamos que la mayoría de los valores de ambas técnicas se encuentran dentro del rango clínicamente aceptable.

PALABRAS CLAVE: Adaptación, adaptación marginal, adaptación interna, aleaciones de cromo, ceras.

Facultad de Odontología, Universidad Central del Ecuador. Quito, Ecuador.

Facultad de Odontología, Universidad Nacional Federico Villareal. Lima, Perú.

Facultad de Odontología, Universidad Católica de Santa María. Arequipa, Perú.

Facultad de Estomatología, Universidad Peruana Cayetano Heredia. Lima, Perú.

Departamento Académico de Odontología Social, Facultad de Estomatología, Universidad Peruana Cayetano Heredia. Lima, Perú.

Departamento Académico de Clínica Estomatológica, Facultad de Estomatología, Universidad Peruana Cayetano Heredia. Lima, Perú.

Cirujano Dentista; ' Especialista en Rehabilitación Oral; ' Magister en Estomatología; d Docente. 


\section{SUMMARY}

New techniques are allowing us to migrate from the technique of lost wax cast by conventional centrifugation (CPCC) to techniques such as lost wax cast by induction (CPCI). Objectives: To compare the marginal and internal discrepancy of cobalt-chromium (Co-Cr) alloy unit copes on a chamfer termination line, made with two techniques: CPCC and CPCI, determining which technique had better marginal and internal adaptation. Material and Methods: $13 \mathrm{Co}-\mathrm{Cr}$ alloy unit copings were fabricated for each technique to be evaluated. Silicon replication was used to evaluate the marginal and internal discrepancies. Each sample was cross-sectioned in the vestibular-palatine and mesio-distal directions, then the cervical, axial and occlusal zones were evaluated with a stereomicroscope. Results: 13 unitary $\mathrm{Co}-\mathrm{Cr}$ alloy metal cops were manufactured for each technique to be evaluated. The silicone replica was used to evaluate the marginal and internal discrepancies, each sample was cross-sectioned in the vestibulo-palatal direction and in the mesio-distal sense, then with a stereomicroscope, the cervical, axial and occlusal areas were evaluated. Conclusions: The assumptions of normality were evaluated with the Shapiro-Wilk test. The statistical analyzes were Student's t test and U Mann-Whitney test. The CPCI obtained a lower marginal and internal discrepancy compared to the CPC, but no statistically significant differences were found $(p>0.05)$ between both techniques. Conclusions: Although in most of the evaluation points the copings made by the CPCI presented better values of marginal and internal adaptation compared to the CPCC, these results are only descriptive values that were not conclusive, since in most of the evaluated points there was no statistically significant difference $(\mathrm{p}>0.05)$. We observed that most of the values of both techniques are within the clinically acceptable range.

\section{KEYWORDS: Adaptation, marginal fit, internal fit, chromium alloys, waxes.}

\section{INTRODUCCIÓN}

La técnica de la cera perdida colado por centrifugación convencional es una de las técnicas que más se usan para la confección de cofias metálicas, utiliza para fundir el metal sopletes que combinan oxigeno con gas natural. Esta técnica está siendo remplazado por la opción del colado por centrifugación por inducción que funde el metal mediante el calor generado por alta frecuencia eléctrica, esto evitaría los posibles errores ya que estos sistemas controlan la temperatura exacta, con el fin de mejorar la adaptación y el tiempo de trabajo (1-5).

Dentro de los diferentes tipos de línea de terminación para corona metalcerámica tenemos: chamfer, hombro-bisel y bisel de $135^{\circ}(6,7)$. La mayoría de autores recomienda la terminación tipo chamfer en coronas metalcerámicas, por que proporciona un adecuado espacio a nivel cervical para el opáquer y la cerámica, una buena adaptación tras el proceso de colado, suficiente resistencia para que la cocción de la porcelana no distorsione esa adaptación y proporciona mejor escurrimiento al agente cementante $(8,9)$.

La adaptación marginal se define como el adecuado ajuste que debe existir entre la línea de terminación de la preparación dentaria y el margen cervical de la restauración, la distancia que existe entre estos dos puntos se conoce como discrepancia marginal. Obtener una buena adaptación marginal es uno de los problemas que con mayor frecuencia se presentan en una prótesis fija ya que al no existir éste, podríamos tener problemas principalmente la microfiltración bacteriana, disolución del cemento y por consiguiente caries secundarias entre otros. La mayoría de autores coincide que la discrepancia marginal no debe ser mayor a $120 \mu \mathrm{m}$ para ser clínicamente aceptable (10-14).

La adaptación interna se define como el ajuste que se da entre la estructura de la restauración y la superficie del pilar dentario, la distancia perpendicular que existe entre estos dos puntos se conoce como discrepancia interna, esta distancia debe ser uniforme para proporcionar un espacio adecuado al agente cementante y así conferir a la restauración la retención y resistencia adecuada. La gran mayoría de autores coincide en que debe ser uniforme pudiendo oscilar en un rango de 50 a $350 \mu \mathrm{m}(15-18)$.

En el 2011 Örtorp et al., realizaron una evaluación in vitro de la adaptación marginal e interna sobre próte- 
sis fijas de Co-Cr en la que utilizaron cuatro técnicas de elaboración: cera perdida colado convencional, sinterizado laser, cera perdida fresado $\mathrm{CAD} / \mathrm{CAM}$ y fresado bloque sinterizado duro. Realizaron un modelo en resina epóxica en la cual hicieron 32 troqueles con sus respectivas prótesis fijas divididos en cuatro grupos. Con la ayuda de un estereomicroscopio midieron el espesor de la película de cemento de la parte marginal e interna, simultáneamente se fueron tomando fotografías digitales con un aumento de $12 \mathrm{X}$ y después se analizaron mediante un software especializado. Hallaron que existe una mejor adaptación con la técnica de sinterizado laser seguido de cera perdida fresado CAD/CAM, cera perdida colado convencional y fresado bloque sinterizado duro. Además, encontraron que la mejor adaptación fue a lo largo de las paredes axiales y en la línea de preparación chamfer. La mayor desadaptación se encontró en la zona oclusal de todas las muestras (16).

En el 2014 Abad et al., evaluaron la adaptación marginal de cofias metálicas fabricadas mediante cuatro técnicas de colado. Evaluaron 15 premolares sanos preparados para recibir una corona completa, se escanearon los modelos y se diseñaron cofias mediante el software CEREC In Lab® (Dentsply Sirona, Bensheim, Alemania). Se fabricaron 60 cofias de Acryl CAD® (Ivoclar Vivadent, Schaan, Liechtenstein), las cuales estuvieron distribuidas en 15 para cada sistema de colado: presión al vacío y colado por inducción de alta frecuencia Nautilus ${ }^{\circledR}$ (BEGO GmbH, Bremen, Alemania), centrifugado eléctrico y colado por inducción Fornax ${ }^{\circledR}$ (BEGO GmbH, Bremen, Alemania), centrifugado eléctrico en la Fundor T® (BEGO $\mathrm{GmbH}$, Bremen, Alemania) y fundida por soplete, y por ultimo centrifugado por cuerda en centrifuga convencional Kerr® (Kerr Corporation, California, USA) y fundida por soplete. Todos los sistemas evaluados mostraron resultados clínicamente aceptables y menores a $120 \mu \mathrm{m}$, pero la técnica de colado por inducción de alta frecuencia y presión al vacío tuvo rangos de discrepancia menores, seguida del colado por inducción y centrifugado eléctrico, las que tuvieron rangos de discrepancia mayores fueron las de centrifugado eléctrico y centrifugado convencional ambas fundidas por soplete (19).

En el 2015 Huang et al., hacen referencia a la Norma ANSI-ADA No. 8 de la Asociación Dental Americana, donde ha establecido "un grosor de película máxi- mo de $25 \mu \mathrm{m}$ para un agente cementante, que corresponde al grosor de película del cemento de fosfato de zinc tipo I", pero que debido a que las discrepancias de ese espesor son difíciles de obtener, la mayoría de los autores aceptan discrepancias mayores (20).

El propósito del presente estudio fue evaluar la adaptación marginal e interna de cofias metálicas unitarias fabricadas con aleación Co-Cr sobre una línea de terminación tipo chamfer comparando dos técnicas: CPCC y CPCI, brindando información valiosa sobre la elección de la técnica que ofreció mejores resultados.

\section{MATERIAL Y MÉTODOS}

Se hizo una preparación dentaria en una pieza natural premolar superior realizando una línea de terminación chamfer con un ángulo de convergencia de $12^{\circ}$, luego se duplicó la preparación dentaria con el CAD/ CAM Ceramill@ (Amann Girrbach AG, Koblach, Germany) creando un modelo maestro de $\mathrm{Co}-\mathrm{Cr}$ Ceramill@ Sintron (Amann Girrbach AG, Koblach, Germany) (figura 1). Se tomaron impresiones con silicona de adición fluida y pesada, se obtuvo modelos de yeso tipo IV, 13 modelos de yeso para la técnica de CPCC y 13 modelos de yeso para la técnica de CPCI. En los modelos de yeso para la técnica de CPCC de acuerdo al procedimiento de confección se aplicó un sellador de poros o endurecedor de $5 \mu \mathrm{m}$ llamado die: master dúo (Renfert $\mathrm{GmbH}$, Hilzingen, Germany), luego se aplicó dos capas del espaciador Pico-Fit (Renfert GmbH, Hilzingen, Germany) de 20 $\mu \mathrm{m}$ a 1 milímetro por encima de la línea de terminación y finalmente una capa de aislante para cera Picosep (Renfert GmbH, Hilzingen, Germany) de $5 \mu \mathrm{m}$, obteniendo las medidas para el espacio del agente cementante de $10 \mu \mathrm{m}$ en la zona cervical y de $50 \mu \mathrm{m}$ en la zona interna. Se enceró la cofia colocando cera cervical roja StarWax C (Dentaurum GmbH, Ispringen, Germany), luego con la ayuda del hotty LED (Renfert $\mathrm{GmbH}$, Hilzingen, Germany) se terminó de encerar las cofias con la cera GEO Dip (Renfert GmbH, Hilzingen, Germany). Después se retoca a nivel cervical con PKT $\mathrm{N}^{\circ} 1$ y cera para modelar crema StarWax D (Dentaurum $\mathrm{GmbH}$, Ispringen, Germany). Se retiró la cofia de cera del modelo y se calibra a $0.5 \mathrm{~mm}$ de espesor. Se vuelve a colocar en el modelo y se readaptó el margen con cera cervical roja StarWax C (Dentaurum $\mathrm{GmbH}$, Ispringen, Germany) utilizando PKT $\mathrm{N}^{\circ} 1$ dejando una banda de $1 \mathrm{~mm}$ de ancho. Se 
colocó un bebedero de cera, se puso la cofia de cera en un cilindro preformado el cual fue llenado con un yeso de revestimiento luego se llevó al horno de precalentamiento rápido para la eliminación de cera por evaporación, se realizó la fundición de las pastillas de aleación Co- Cr CROMORON Premium C (DG DENTAL d.o.o, Gornji Grad, Slovenia) con ayuda de un soplete y se impulsó la aleación metálica al interior de la cámara de moldeado mediante la acción de una fuerza centrífuga. Para la técnica de CPCI se realizó el mismo procedimiento de encerado, revestido y eliminación de cera anteriormente descrito pero, la diferencia es que no se utilizó el soplete para fundir el metal, esta técnica utilizó para fundir la aleación de Co-Cr Cromoron Premium C (DG Dental d.o.o, Gornji Grad, Slovenia) la máquina centrífuga compacta para colado con mecanismo de fusión por inducción eléctrica de gran potencia Fornax ${ }^{\circledR} \mathrm{T}(\mathrm{BEGO} \mathrm{GmbH}$, Bremen, Alemania) de 3600 VA y refrigeración inte-

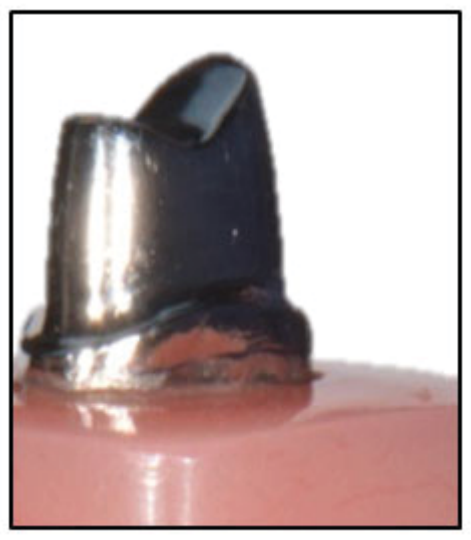

Figura 1. Modelo maestro

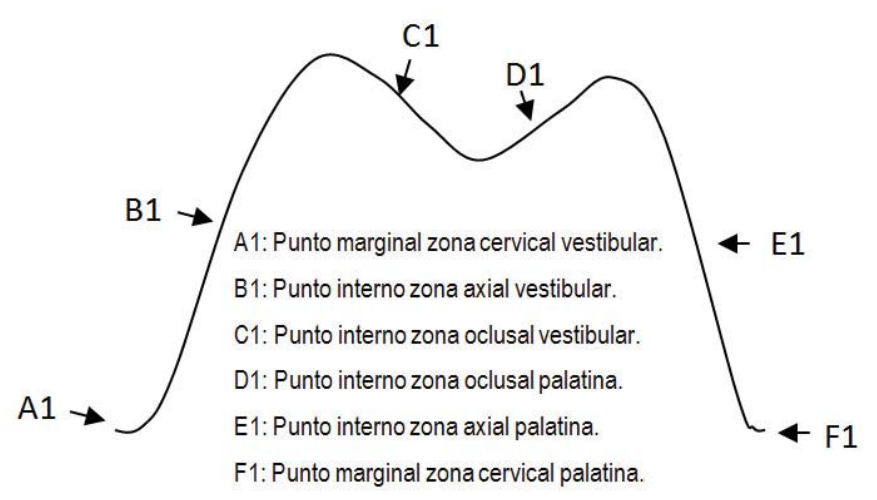

Figura 3. Corte vestíbulo-palatino grada correctamente calibrada; se precalentó el metal en la máquina durante $15 \mathrm{~s}$, se introdujo el cilindro de yeso refractario que salió del horno de precalentamiento, se configuró la máquina de inducción para que suba la temperatura a $1400^{\circ} \mathrm{C}$, luego de aproximadamente $25 \mathrm{~s}$ se observó que la aleación cambió de su estado sólido a un estado líquido, en ese momento se activó la centrifuga para que la aleación se introduzca en el cilindro por acción de la fuerza centrífuga.

Una vez que los colados ha enfriado a temperatura ambiente se procedió al desrevestido, se arenaron las cofias con polvo de óxido de aluminio con el objetivo de limpiar el yeso adherido a la superficie del colado. Las cofias de Co-Cr fueron agrupadas en 13 cofias por grupo, la adaptación marginal e interna fue evaluada con la técnica de réplica de silicona VPES FitChecker ${ }^{\mathrm{TM}}$ Advance (GC Corporation, Tokyo, Japan), esta se inyecto dentro de las cofias y se asentó sobre el

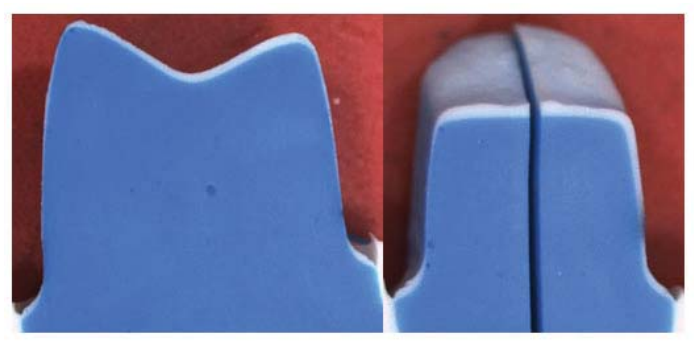

Figura 2. Modelo maestro

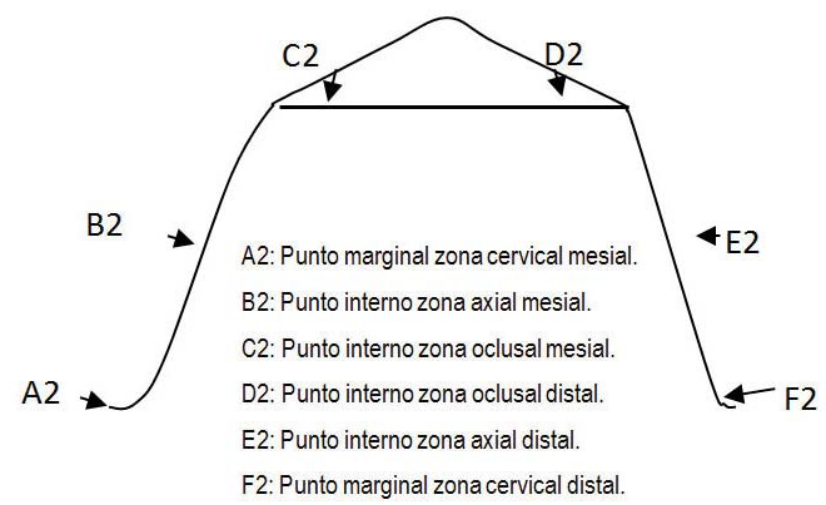

Figura 4. Corte mesio-distal 
modelo maestro de Co-Cr a una fuerza de $15 \mathrm{~N}$ durante dos minutos con una máquina de ensayo universal Instron CTM-5L (LG Electronics, Seúl, Korea). Se retiró la cofia del modelo maestro y se inyectó sobre la VPES silicona fluida de adición obteniendo la réplica de silicona, se realizaron dos cortes en sentido vestíbulo-palatino y mesio-distal (figura 2). La numeración "1" correspondió al corte en sentido vestíbulo-palatino donde el punto A1 fue el punto marginal vestibular y F1 fue el punto marginal palatino, B1 y E1 representaron las zonas axiales, C1 y D1 la zona oclusal. La numeración "2" correspondió al corte en sentido mesio-distal donde el punto A2 fue el punto marginal mesial y F2 fue el punto marginal distal, B2 y E2 representaron las zonas axiales, C2 y D2 las zonas oclusales (figura 3 y figura 4). Se evaluó las discrepancias marginales e internas en un estéreomicroscopio Greenough Leica S8 APO (Leica Biosystems $\mathrm{GmbH}$, Wetzlar, Germany) a un aumento de 40X con el software Leica Application Suite LAS v3.4 (Leica Biosystems GmbH, Wetzlar, Germany) medidos en micrómetros $(\mu \mathrm{m})$. Se utilizó el programa STATA versión 12 para el análisis univariado y bivariado, donde se evaluaron los supuestos de normalidad con la prueba de Shapiro-Wilk. Se empleó la prueba $\mathrm{t}$ de Student para distribución normal y la prueba $\mathrm{U}$ Mann-Whitney si no se presentó distribución normal. El presente estudio utilizó un nivel de confianza al $90 \%(\mathrm{p}<0,05)$.

\section{RESULTADOS}

En la tabla 1 se muestran resultados descriptivos de la discrepancia marginal de las cofias obtenidas con la técnica CPCC según tipo de corte y punto marginal, donde se encontraron valores promedios menores de discrepancias marginales en el corte vestíbulo-palatino en los puntos A1 con $89,65 \pm 58,39 \mu \mathrm{m}$ y F1 con $92 \pm$ $88,40 \mu \mathrm{m}$, en comparación con los valores promedio en las medidas del corte mesio-distal en los puntos A2 con $91,72 \pm 67,13 \mu \mathrm{m}$ y F2 con $97,66 \pm 50,59 \mu \mathrm{m}$.

En la tabla 2 se muestran los resultados descriptivos de la discrepancia interna obtenidos con la técnica de CPCC según tipo de corte, zona y punto interno, los valores promedio en la zona axial en el corte vestíbulo-palatino en el punto B1 de $60,09 \pm 22,66 \mu \mathrm{m}$ y

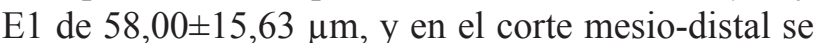
registró una menor discrepancia interna en el punto

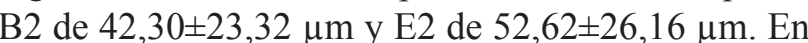
la zona oclusal en el corte vestíbulo-palatino registró una menor discrepancia interna en los puntos $\mathrm{C} 1$ de $162,67 \pm 53,38 \mu \mathrm{m}$ y D1 $195,92 \pm 69,47 \mu \mathrm{m}$, en comparación del corte mesio-distal en los puntos $\mathrm{C} 2$ de $209,19 \pm 55,71 \mu \mathrm{m}$ y D2 de $203,78 \pm 62,94 \mu \mathrm{m}$. Las menores discrepancias internas en ambos cortes mesio-distal y vestíbulo-palatino fueron en la zona axial en comparación a la zona oclusal.

Tabla 1. Discrepancia marginal de las cofias metálicas unitarias de aleación Co-Cr sobre una línea de terminación tipo chamfer realizada con la técnica de cera perdida colada por centrifugación convencional, según tipo de corte y punto marginal.

\begin{tabular}{lcclllll}
\hline Tipo de corte & $\begin{array}{c}\text { Punto } \\
\text { Marginal }\end{array}$ & Min & Max & Promedio & D.E. & Mediana & DIC \\
\hline \multirow{2}{*}{ Vestíbulo Palatino } & A1 & 32,77 & 219,94 & 89,65 & 58,39 & 57,93 & 38,04 \\
& F1 & 26,18 & 296,2 & 92,00 & 88,40 & 56,92 & 30,48 \\
Mesio-Distal & A2 & 23,02 & 224,83 & 91,72 & 67,13 & 63,5 & 44,76 \\
& F2 & 38,62 & 202,63 & 97,66 & 50,59 & 84,42 & 32,39 \\
\hline
\end{tabular}


Tabla 2. Discrepancia interna de las cofias metálicas unitarias de aleación Co-Cr sobre una línea de terminación tipo chamfer realizada con la técnica de cera perdida colada por centrifugación convencional, según tipo de corte, zona y punto interno.

\begin{tabular}{ccccccccc}
\hline $\begin{array}{c}\text { Tipo de } \\
\text { corte }\end{array}$ & $\begin{array}{c}\text { Zona } \\
\text { interna }\end{array}$ & $\begin{array}{c}\text { Punto } \\
\text { Interno }\end{array}$ & Min & Max & Promedio & D.E. & Mediana & DIC \\
\hline \multirow{2}{*}{$\begin{array}{c}\text { Vestibulo } \\
\text { palatino }\end{array}$} & Axial & B1 & 29,32 & 100,43 & 60,09 & 22,66 & 54,61 & 14,43 \\
& & E1 & 35,42 & 79,57 & 58,00 & 15,63 & 61,03 & 21,24 \\
& Oclusal & C1 & 90,56 & 280,50 & 162,67 & 53,38 & 161,45 & 34,14 \\
& & D1 & 115,30 & 331,73 & 195,92 & 69,47 & 166,99 & 73,99 \\
Mesio & Axial & B2 & 11,43 & 80,17 & 42,30 & 23,32 & 35,76 & 27,92 \\
Distal & & E2 & 24,26 & 114,87 & 52,62 & 26,16 & 44,45 & 22,71 \\
& \multirow{2}{*}{ Oclusal } & C2 & 119,41 & 296,00 & 209,19 & 55,71 & 201,18 & 88,94 \\
& & D2 & 120,66 & 304,41 & 203,78 & 62,94 & 195,59 & 80,75
\end{tabular}

Tabla 3. Discrepancia marginal de las cofias metálicas unitarias de aleación Co-Cr sobre una línea de terminación tipo chamfer realizada con la técnica de cera perdida colada por centrifugación por inducción, según tipo de corte y punto marginal.

\begin{tabular}{llllllll}
\hline Tipo de corte & $\begin{array}{l}\text { Punto } \\
\text { Marginal }\end{array}$ & Min & Max & Promedio & D.E. & Mediana & DIC \\
\hline $\begin{array}{l}\text { Vestibulo- } \\
\text { Palatino }\end{array}$ & A1 & 35,03 & 116,27 & 64,87 & 27,36 & 64,21 & 21,49 \\
& F1 & 37,89 & 102,73 & 72,13 & 22,62 & 70,13 & 21,38 \\
Mesio-Distal & A2 & 54,15 & 124,47 & 93,43 & 23,89 & 101,70 & 17,71 \\
& F2 & 26,18 & 125,07 & 78,66 & 30,47 & 69,29 & 21,30 \\
\hline
\end{tabular}

Tabla 4. Discrepancia interna de las cofias metálicas unitarias de aleación Co-Cr sobre una línea de terminación tipo chamfer realizada con la técnica de cera perdida colada por centrifugación por inducción, según tipo de corte, zona y punto interno.

\begin{tabular}{ccccccccc}
\hline $\begin{array}{c}\text { Tipo de } \\
\text { corte }\end{array}$ & $\begin{array}{c}\text { Zona } \\
\text { interna }\end{array}$ & $\begin{array}{c}\text { Punto } \\
\text { Interno }\end{array}$ & Min & Max & Promedio & D.E. & Mediana & DIC \\
\hline \multirow{3}{*}{$\begin{array}{c}\text { Vestibulo } \\
\text { palatino }\end{array}$} & \multirow{2}{*}{ Axial } & B1 & 15,40 & 99,19 & 54,29 & 24,92 & 60,24 & 12,50 \\
& oclusal & E1 & 17,60 & 87,20 & 54,29 & 18,29 & 57,43 & 10,43 \\
& & D1 & 111,38 & 223,14 & 156,89 & 31,27 & 159,14 & 21,22 \\
\multirow{2}{*}{ Mesio } & \multirow{2}{*}{ Axial } & B2 & 24,12 & 76,09 & 37,98 & 11,47 & 34,29 & 4,15 \\
Distal & \multirow{2}{*}{ oclusal } & E2 & 22,91 & 76,41 & 43,10 & 14,38 & 40,51 & 8,98 \\
& & D2 & 115,64 & 210,89 & 173,33 & 25,56 & 174,00 & 13,98 \\
& & 146,11 & 223,29 & 172,93 & 23,10 & 163,68 & 12,53 \\
\hline
\end{tabular}


Tabla 5. Comparación de la adaptación marginal en cofias metálicas unitarias de aleación Co-Cr sobre una línea de terminación tipo chamfer realizadas por técnica de la cera perdida colada por centrifugación: convencional y por inducción, según tipo de corte y punto marginal.

\begin{tabular}{cccccc}
\hline Tipo de corte & $\begin{array}{c}\text { Punto } \\
\text { Marginal }\end{array}$ & $\begin{array}{c}\text { Centrifugación } \\
\text { convencional }\end{array}$ & $\begin{array}{c}\text { Centrifugación } \\
\text { por inducción }\end{array}$ & $\begin{array}{c}\text { Diferencia de } \\
\text { medias }\end{array}$ & valor de p \\
\hline $\begin{array}{c}\text { Vestíbulo- } \\
\text { palatino }\end{array}$ & A1 & 89,65 & 64,87 & 24,78 & $0,4887 * *$ \\
& F1 & 92,00 & 72,13 & 19,86 & $0,3695 * *$ \\
$\begin{array}{c}\text { Mesio- } \\
\text { Distal }\end{array}$ & A2 & 91,72 & 93,43 & $-1,70$ & $0,2702 * *$ \\
\hline $\begin{array}{c}* \text { Prueba t student } \\
* * \text { U Mann-Whitney }\end{array}$ & & 78,66 & 78,66 & 19,01 & $0,2574 *$ \\
\end{tabular}

Tabla 6. Comparación de la adaptación interna en cofias metálicas unitarias de aleación Co-Cr sobre una línea de terminación tipo chamfer realizadas por técnica de la cera perdida colada por centrifugación: convencional y por inducción, según tipo de corte, zona y punto interno.

\begin{tabular}{lccccccc}
\hline $\begin{array}{c}\text { Tipo de } \\
\text { corte }\end{array}$ & $\begin{array}{c}\text { Zona } \\
\text { interna }\end{array}$ & $\begin{array}{c}\text { Punto } \\
\text { interno }\end{array}$ & $\begin{array}{c}\text { Centrifugación } \\
\text { convencional }\end{array}$ & $\begin{array}{c}\text { Centrifugación } \\
\text { por inducción }\end{array}$ & $\begin{array}{c}\text { Diferencia } \\
\text { de medias }\end{array}$ & $\begin{array}{c}\text { valor } \\
\text { de p }\end{array}$ & \\
\hline \multirow{2}{*}{$\begin{array}{l}\text { Vestíbulo } \\
\text { palatino }\end{array}$} & Axial & B1 & 60,09 & 54,29 & 5,80 & 0,5407 & $*$ \\
& & E1 & 58,00 & 54,29 & 3,50 & 0,6043 & $*$ \\
& oclusal & C1 & 162,67 & 133,04 & 29,63 & 0,0892 & $*$ \\
& & D1 & 195,92 & 156,89 & 39,02 & 0,1439 & $* *$ \\
Mesio & Axial & B2 & 42,30 & 39,98 & 4,32 & 0,5546 & $*$ \\
Distal & & E2 & 52,62 & 43,10 & 9,52 & 0,2615 & $*$ \\
& oclusal & C2 & 209,19 & 173,33 & 35,87 & 0,0455 & $*$ \\
& & D2 & 203,78 & 172,93 & 30,85 & 0,1101 & $*$ \\
\hline
\end{tabular}

** U Mann-Whitney

significancia $\mathrm{p}<0,05$

La tabla 3 muestra la discrepancia marginal de las cofias obtenidas con la técnica CPCI según tipo de corte y punto marginal, en el corte vestíbulo-palatino se obtuvo resultados promedios en el punto A1 de $64,87 \pm 27,36 \mu \mathrm{m}$ y en el punto $\mathrm{F} 1 \mathrm{de} 72,13 \pm 22,62$ $\mu \mathrm{m}$, en el corte mesio-distal se obtuvo resultados promedios en los puntos A2 de 93,43 $\pm 23,89 \mu \mathrm{m}$ y F2 de $78,66 \pm 30,47 \mu \mathrm{m}$. Se observó que el punto que presenta menor discrepancia marginal en sentido vestíbulo-palatino es el A1, y en sentido mesio-distal es el punto F2.
La tabla 4 muestra la discrepancia interna de las cofias obtenidas con la técnica CPCI, mostrando que los puntos de la zona axial, tanto en el corte vestíbulo-palatino como en el corte mesio-distal (B1:54,29 $\pm 24,92$ $\mu \mathrm{m}, \quad \mathrm{E} 1: 54,29 \pm 18,29 \mu \mathrm{m}, \quad \mathrm{B} 2: 37,98 \pm 11,47 \mu \mathrm{m}$, $\mathrm{E} 2: 43,10 \pm 14,38 \mu \mathrm{m})$, presentan una menor discrepancia interna con respecto a los puntos de la zona oclusal $(\mathrm{C} 1: 133,04 \pm 28,09 \mu \mathrm{m}, \mathrm{D} 1: 156,89 \pm 31,27 \mu \mathrm{m}$, $\mathrm{C} 2: 173,33 \pm 25,56 \mu \mathrm{m}, \mathrm{D} 2: 172,93 \pm 23,10 \mu \mathrm{m})$. En el corte en sentido vestíbulo-palatino se encontró una discrepancia axial menor en el punto $\mathrm{B} 1 \mathrm{y}$ una discre- 
pancia oclusal menor en el punto $\mathrm{C} 1 \mathrm{y}$ en sentido mesio-distal se encontró una discrepancia axial menor en B2 y una discrepancia oclusal menor en el punto D2. La tabla 5 se comparó las adaptaciones marginales entre las dos técnicas, podemos observar que en los puntos A1, F1 y F2 existe una mejor adaptación marginal en la técnica CPCI en comparación con la técnica CPCC; mientras que en el punto A2 la mejor adaptación marginal se observó en la técnica CPCC; pero sólo son valores descriptivos que no fueron concluyentes porque no se encontró diferencia estadísticamente significativa entre las dos técnicas evaluadas $(\mathrm{p}>0,05)$. La tabla 6 se comparó las adaptaciones internas entre las dos técnicas, en el corte mesio-distal, en la zona axial con la técnica CPCC, se encontró en el punto B2 el menor valor promedio de $42,30 \mu \mathrm{m}$ y en el mismo punto para la técnica CPCI se encontró el menor valor promedio de $39,98 \mu \mathrm{m}$, pero no hubo diferencia estadísticamente significativa. En el corte vestíbulo-palatino, en la zona oclusal para la técnica CPCC, se encontró a nivel del punto C1 el menor valor promedio de $162,67 \mu \mathrm{m}$ y en el mismo punto para la técnica CPCI se encontró el menor valor promedio de $133,04 \mu \mathrm{m}$, pero no hubo diferencia estadísticamente significativa. Solo en el punto $\mathrm{C} 2$ se obtuvo un valor de $p=0,0455$ siendo estadísticamente significativo a nivel de este punto de medición para la técnica CPCI de 173,33 $\mu \mathrm{m}$ en comparación con la técnica CPCC de 209,19 $\mu \mathrm{m}$, para los demás puntos de medición no existe diferencia estadísticamente significativa en la adaptación interna entre ambas técnicas $(\mathrm{p}>0,05)$. En ambas técnicas la zona axial tuvo mejor adaptación interna que la zona oclusal.

\section{DISCUSIÓN}

El presente estudió fue de tipo experimental y transversal, evaluó la adaptación marginal e interna de cofias metálicas unitarias de aleación de $\mathrm{Co}-\mathrm{Cr}$, fabricadas con dos técnicas: CPCC y CPCI.

La adaptación marginal de una restauración protésica es uno de los criterios más importantes para el éxito a largo plazo de la misma. Lograr un correcto asentamiento de la restauración a la preparación es un proceso complejo y prolijo que involucra varios factores como: la preparación, integridad de la línea de terminación, la técnica de elaboración, manipulación y experiencia del técnico y finalmente pero no menos importante el cemento, por todos estos facto- res algunos trabajos a veces requieren ser repetidos y la principal causa es que no se observa una correcta adaptación marginal (21-28).

Abad et al., evaluaron la adaptación marginal de cofias metálicas $\mathrm{Ni}-\mathrm{Cr}$ fabricadas mediante cuatro técnicas de colado: presión al vacío con colado por inducción de alta frecuencia, centrifugado eléctrico con colado por inducción, centrifugado eléctrico con fundición por soplete y por ultimo centrifugado por cuerda en centrifuga convencional con fundición por soplete. La técnica de presión al vacío con colado por inducción de alta frecuencia tuvo rangos de discrepancia menores $(59,26 \mu \mathrm{m})$, seguida del centrifugado eléctrico con colado por inducción $(63,6 \mu \mathrm{m})$, las que tuvieron rangos de discrepancia mayores fueron las de centrifugado eléctrico $(63,8 \mu \mathrm{m})$ y centrifugado convencional $(69,08 \mu \mathrm{m})$ ambas fundidas por soplete.

La aleación de Co-Cr utilizada en el presente estudio difiere con la aleación utilizada de $\mathrm{Ni}$-Cr del estudio de Abad et al., ambos estudios coinciden en que hay mejor adaptación marginal cuando se utiliza la técnica CPCI (19).

En el estudio que realizó Pulido et al., cuyo objetivo fue comparar la adaptación marginal e interna de dos aleaciones: $\mathrm{Co}-\mathrm{Cr}$ y $\mathrm{Ni}-\mathrm{Cr}$, coladas por inducción eléctrica en la máquina Fornax ${ }^{\circledR}$ T (BEGO GmbH, Bremen, Alemania) encontró que la adaptación marginal del Co-Cr fue mejor $(53,31 \mu \mathrm{m})$ comparada con la de Ní-Cr $(84,39 \mu \mathrm{m})$ pero sin diferencia estadísticamente significativa.

Cuando comparó la adaptación interna en la zona axial encontró que la aleación $\mathrm{Co}-\mathrm{Cr}(4,96 \mu \mathrm{m})$ presentó mejor adaptación que la de $\mathrm{Ni}-\mathrm{Cr}(48,22$ $\mu \mathrm{m})$ con diferencia estadísticamente significativa y en la zona oclusal encontró que la aleación de $\mathrm{Co}-\mathrm{Cr}$ $(45,84 \mu \mathrm{m})$ presentó una mejor adaptación que la Ni$\mathrm{Cr}(105,11 \mu \mathrm{m})$ pero sin diferencia estadísticamente significativa. Comparando estos resultados con el presente estudio, nosotros utilizamos aleaciones de $\mathrm{Co}-\mathrm{Cr}$ encontrando mejor adaptación marginal en la mayoría de los puntos evaluados con la técnica de cera pérdida colado por inducción comparándola con la de colado convencional, pero sin diferencia estadísticamente significativa. Con respecto a la adaptación interna se encontró mejor adaptación en la zona axial comparándola con la zona oclusal, pero 
sin diferencia estadísticamente significativa en la adaptación interna entre ambas técnicas (27).

Estos resultados contrastan con los de Cogolludo et al., quienes evaluaron cofias elaboradas con tres técnicas: colado centrifugado convencional, colado centrifugado por inducción y colado por inducción y presión de vacío, concluyeron que la técnica de colado centrifugado convencional obtuvo los menores valores de discrepancia marginal, resaltan que un correcto procedimiento de esta técnica puede superar la adaptación marginal obtenidas con otras técnicas aunque el estudio fue realizado con aleaciones de $\mathrm{Ni}$ Cry aleaciones de Pd- Cr (29).

De acuerdo a los resultados obtenidos a pesar que encontramos mejores valores en la adaptación marginal en las cofias unitarias de $\mathrm{Co}-\mathrm{Cr}$ obtenidas mediante la técnica CPCI en la mayoría de puntos evaluados (A1: 64,87 $\mu \mathrm{m}, \mathrm{F} 1: 72,13 \mu \mathrm{m}, \mathrm{F} 2: 78,66 \mu \mathrm{m}$,) comparándola con la técnica CPCC (A1: 89,65 $\mu \mathrm{m}, \mathrm{F} 1: 92,00$ $\mu \mathrm{m}, \mathrm{F} 2: 97,66 \mu \mathrm{m})$, estos valores solo fueron descriptivos pero no concluyentes por no tener una diferencia estadísticamente significativa, en ambas técnicas los promedios fueron menores a las $120 \mu \mathrm{m}$ que es el límite máximo del rango estándar clínicamente aceptable. Con respecto a la adaptación interna tanto en sentido vestíbulo-palatino y mesio-distal la técnica CPCI presentó mejores valores de adaptación interna en comparación con la técnica CPCC, en ambas técnicas la zona axial tuvo mejor adaptación interna que la zona oclusal, pero estos resultados sólo son valores descriptivos que no fueron concluyentes, ya que en la mayoría de los puntos evaluados no existió diferencia estadísticamente significativa. Por lo tanto, los resultados no fueron concluyentes como para indicar que técnica logró la mejor adaptación marginal e interna.

Los puntos de referencia para realizar las mediciones de la adaptación marginal varían mucho entre los investigadores y varios estudios llegan a la conclusión basada en su propio criterio (17). En el presente estudio se realizaron medidas de la adaptación marginal e interna en doce puntos distribuidos en la zona vestibular, palatina, mesial y distal de cofias metálicas de Co-Cr obtenidas mediante las técnicas CPCC y CPCI.

Nuestro estudio difiere del estudio realizado por Groten et al., en donde relataron que se debía medir como mínimo cincuenta puntos en la terminación marginal de una cofia (30), ellos tomaron un numero muestral de 10 coronas, aunque este número de medidas en la revisión sistemática de Contrepois et al., concluyeron que dieciocho puntos de medición fueron necesarios para evaluar coronas experimentales hechas en un laboratorio (31). Holmes et al., y Pera et al., midieron sus muestras en cuatro puntos marginales $(32,33)$.

Como podemos observar con los datos referenciales y los de este estudio hay una marcada variabilidad en las discrepancias obtenidas entre las dos técnicas de fabricación o elaboración de cofias metálicas, esto puede deberse a que la adaptación de las cofias es muy dependiente del factor humano y que eso depende de la habilidad del técnico del laboratorio para obtener óptimos resultados.

Se recomienda realizar investigaciones que permitan evaluar la adaptación marginal e interna mediante otras técnicas de fabricación, después del proceso de aplicación de la porcelana, con diferente línea de terminación, con diferente ángulo de convergencia, con otros materiales de fabricación de cofias, etc.

\section{CONCLUSIONES}

$\mathrm{Al}$ evaluar la adaptación marginal e interna no hubo una diferencia estadísticamente significativa entre ambos grupos. A pesar de que los valores descriptivos de la adaptación marginal de las cofias confeccionadas mediante la técnica de CPCI fue mejor que la técnica de la CPCC y en la adaptación interna los resultados obtenidos fueron similares para ambas técnicas.

\section{Correspondencia:}

Silvio Requena Cisneros

Correo electrónico: silvinrequena@hotmail.com

\section{REFERENCIAS BIBLIOGRÁFICAS}

1. Pettenò D, Schierano G, Bassi F, Bresciano ME, Carossa S. Comparison of marginal fit of 3 different metal-ceramic systems: an in vitro study. Int J Prosthodont. 2000; 13(5):405-8.

2. Anusavice KJ, Phillips RW, Shen C, Rawls HR. Phillips' Science of Dental Materials. 12th ed. St. Louis: Elsevier/Saunders; 2013.

3. White SN, Sorensen JA, Kang SK, Caputo AA. Microleakage of a new crown and fixed partial denture luting agent. J Prosthet Dent. 1992; 67(2):156-61.

4. Hunter AJ, Hunter AR. Gingival margins for crowns: 
a review and discussion. Part II. Discrepancies and configurations. J Prosthet Dent. 1990; 64(6):636-42.

5. Darvell BW. A glossary of terms for dental materials science. 12th ed. India: Woodhead Publisher; 2012.

6. Syu JZ, Byrne G, Laub LW, Land MF. Influence of finish-line geometry on the fit of crowns. Int J Prosthodont. 1993; 6(1):25-30.

7. Bottino MA, Valandro LF, Buso L, Ozcan M. The influence of cervical finish line, internal relief, and cement type on the cervical adaptation of metal crowns. Quintessence Int. 2007; 38(7):425-32.

8. Piwowarczyk A, Lauer HC, Sorensen JA. Microleakage of various cementing agents for full cast crowns. Dent Mater. 2005; 21(5):445-53.

9. Reitemeier B, Hansel K, Kastner C, Walter MH. Metal-ceramic failure in noble metal crowns: 7-year results of a prospective clinical trial in private practices. Int J Prosthodont. 2006; 19(4):397-9.

10. Roberts HW, Berzins DW, Moore BK, Charlton DG. Metal-ceramic alloys in dentistry: a review. J Prosthodont. 2009; 18(2):188-94.

11. Kurbad A, Schnock HA. A method for the easy fabrication of all-ceramic bridges with the Cerec system. Int J Comput Dent. 2009; 12(2):171-185.

12. The glossary of prosthodontic terms. J Prosthet Dent. 2005; 94:63.

13. Felton DA, Kanoy BE, Bayne SC, Wirthman GP. Effect of in vivo crown margin discrepancies on periodontal health. J Prosthet Dent. 1991; 65(3):357-64.

14. Christensen GJ. Marginal fit of gold inlay castings. J Prosthet Dent. 1966; 16(2):297-305.

15. Ishikiriama A, Oliveira J de F, Vieira DF, Mondelli J. Influence of some factors on the fit of cemented crowns. J Prosthet Dent. 1981; 45(4):400-4.

16. Örtorp A, Jönsson D, Mouhsen A, Vult von Steyern P. The fit of cobalt-chromium three-unit fixed dental prostheses fabricated with four different techniques: A comparative in vitro study. Dent Mater. 2011; 27(4):356-63.

17. Quante K, Ludwing K, Kern M. Marginal and internal fit of metal-ceramic crowns fabricated with a new laser melting technology. Dent Mater. 2008; 24 (10):1311-5.

18. Holmes JR, Bayne SC, Holland GA, Sulik WD. Considerations in measurement of marginal fit. J Prosthet Dent. 1989; 62(4):405-8.

19. Abad J, Castro M, Durand E. Comparación de la adaptación marginal de cofias elaboradas en cuatro tipos de máquinas de colado. Revista Odontos. 2014; 43(4): 7-14.

20. Huang Z, Zhang L, Zhu J, Zhang X. Clinical marginal and internal fit of metal ceramic crowns fabricated with a selective laser melting technology. J Prosthet Dent. 2015; 113(6):623-7

21. Nesse H, Mari D, Myhre M, Øilo M. Internal and mar- ginal fit of cobalt-chromium fixed dental prostheses fabricated with three different techniques. J Prosthet Dent. 2015; 114(5):686-92.

22. Morris HF, Manz M, Stoffer W, Weir D. Casting alloys: the materials and "The Clinical Effects". Adv Dent Res. 1992; 6:28-31.

23. Vojdani M, Torabi K, Farjood E, Khaledi A. Comparison the Marginal and Internal Fit of Metal Copings Cast from Wax Patterns Fabricated by CAD/CAM and Conventional Wax up Techniques. J Dent (Shiraz). 2013; 14(3):118-29.

24. Geis-Gerstorfer J, Schille Ch, Schweizer E, Noack F. Hoffmann R. Comparison of the biocompatibility and corrosion properties of a $\mathrm{CoCr}$ sinter alloy with a casting alloy. Dental dialogue. 2013; 14:20-5.

25. Lombardo G, Nishioka R, Souza R, Michida S, Kojima A, Mesquita A, Buso L. Influence of surface treatment on the shear bond strength of ceramics fused to cobalt-chromium. J Prosthodont. 2010; 19(2):103-11.

26. Schwartz I. A review of methods and techniques to improve the fit of cast restorations. J Prosthet Dent 1986; 56:279-283.

27. Pulido F, Guerra c, Sánchez J, Giraldo H. Comparación de la adaptación marginal e interna de cofias coladas en dos sistemas de aleaciones. Revista Colombiana de Investigación en Odontología. 2014; 5 (15): $147-156$.

28. Villegas AM, Naranjo E, Gómez DM. Pruebas de biocompatibilidad de los materiales de uso odontológico: Revisión de la literatura. Rev Estomat. 2008; 16(2):38-44.

29. Cogolludo PG, Suarez MJ, Peláez J, Lozano JF. Influence of melting and casting methods and finish line design on the marginal discrepancy of nickel-chromium-titanium alloy crowns. Int J Prosthodont. 2010; 23(5):443-5.

30. Groten M, Axmann D, Pröbster L, Weber H. Determination of the minimum number of marginal gap measurements required for practical in-vitro testing. J Prosthet Dent. 2000; 83(1):40-9.

31. Contrepois M, Soenen A, Bartala M, Laviole O. Marginal adaptation of ceramic crowns: a systematic review. J Prosthet Dent. 2013; 110(6):447-454.e10.

32. Holmes JR, Sulik WD, Holland GA, Bayne SC. Marginal fit of castable ceramic crowns. J Prosthet Dent 1992; 67(5):594-599.

33. Pera P, Gilodi S, Bassi F, Carossa S. In vitro marginal adaptation of alumina porcelain ceramic crowns. $\mathrm{J}$ Prosthet Dent 1994; 72(6):585-590.

Recibido: 23-08-2018

Aceptado: 29-11-2018 